ARTICLE

\title{
Adaptive carbon export response to warming in the Sargasso Sea
}

\author{
Michael W. Lomas (iD) ${ }^{1 凶}$, Nicholas R. Bates (D) ${ }^{2,3}$, Rodney J. Johnson ${ }^{2}$, Deborah K. Steinberg (i) ${ }^{4} \&$
}

Tatsuro Tanioka (iD ${ }^{5}$

Ocean ecosystem models predict that warming and increased surface ocean stratification will trigger a series of ecosystem events, reducing the biological export of particulate carbon to the ocean interior. We present a nearly three-decade time series from the open ocean that documents a biological response to ocean warming and nutrient reductions wherein particulate carbon export is maintained, counter to expectations. Carbon export is maintained through a combination of phytoplankton community change to favor cyanobacteria with high cellular carbon-to-phosphorus ratios and enhanced shallow phosphorus recycling leading to increased nutrient use efficiency. These results suggest that surface ocean ecosystems may be more responsive and adapt more rapidly to changes in the hydrographic system than is currently envisioned in earth ecosystem models, with positive consequences for ocean carbon uptake.

\footnotetext{
${ }^{1}$ Bigelow Laboratory for Ocean Sciences, East Boothbay, ME, USA. ${ }^{2}$ Bermuda Institute for Ocean Sciences, St. Georges, Bermuda. ${ }^{3}$ Department of Ocean and Earth Science, University of Southampton, Southampton, UK. ${ }^{4}$ Virginia Institute of Marine Science, William \& Mary, Gloucester Pt., Virginia, VA, USA.

${ }^{5}$ Department of Earth System Science, University of California, Irvine, CA, USA. ${ }^{凶}$ email: mlomas@bigelow.org
} 
P hytoplankton play a central role in regulating global ocean biogeochemical cycles and production in marine food webs ${ }^{1-4}$. Concerns about the impact of upper-ocean warming and increased stratification on phytoplankton production and carbon export have grown during the last decade ${ }^{5-7}$, with observations of synchronous increases in surface ocean temperature and an apparent global decrease of phytoplankton biomass, inferred from changes in chlorophyll a (Chl a) concentration ${ }^{3,4}$. Upper-ocean warming is thought to positively influence phytoplankton primary production by impacting their photosynthetic metabolism ${ }^{8}$, and has a negative effect by increasing stratification leading to reductions in vertical nutrient inputs and subsequent nutrient limitation ${ }^{9}$. Warming of the upper ocean is also predicted to reduce the magnitude of biological carbon export to the ocean interior by reducing upwelling of nutrients and/or by shifts in phytoplankton community composition toward smaller, less dense picophytoplankton $7,10,11$.

The magnitude of this predicted series of changes in marine biogeochemistry is in part dependent upon the stoichiometric relationship between the major macronutrients carbon (C), nitrogen $(\mathrm{N})$, and phosphorus $(\mathrm{P})$, with the canonical Redfield ratio set at $106 \mathrm{C}: 16 \mathrm{~N}: 1 \mathrm{P}^{12,13}$. Fixed stoichiometric models suggest that global net primary production (NPP) may decline by up to $20 \%$ (ref. ${ }^{5}$ ), whereas flexible stoichiometric models indicate $\mathrm{a}<10 \%$ reduction in $\mathrm{NPP}^{7}$. The Arctic Ocean and subtropical gyres appear the most sensitive global ocean regions in both models, with decreases in NPP exceeding the global mean from the models. These NPP reductions, in the models, appear to be driven by a greater decline in phytoplankton growth rate than in phytoplankton biomass stocks, supporting the general view of bottom-up (i.e., nutrient, temperature, light) controls on NPP. As with NPP, flexible stoichiometric models predict smaller decreases in the magnitude of the biological carbon pump due to modeled increases in nutrient use efficiency associated with the flexible stoichiometry of phytoplankton ${ }^{14}$. In terms of reducing the impact of nutrient limitation on carbon export, the benefits of flexible stoichiometry are partially negated by the reduction in mean phytoplankton cell size, which, in the models, also limits the export of carbon associated with smaller phytoplankton. Resolution of these competing processes impacting phytoplanktonmediated processes based upon in situ data is lacking. For example, in the Sargasso Sea, the resident phytoplankton community has a highly flexible macronutrient stoichiometry ${ }^{15,16}$. The cyanobacteria genera Synechococcus and Prochlorococcus contribute substantially to particulate carbon export ${ }^{17}$, resulting in an overall efficient carbon export system ${ }^{18}$.

In the Sargasso Sea at the Bermuda Atlantic Time-series Study (BATS) site, we have quantified relationships between phytoplankton production, nutrients, ocean physics, and carbon export for three decades. Recent studies have confirmed an inverse relationship between temperature and $\mathrm{NPP}^{19}$. Here, we continue to analyze long-term patterns in the coupling of NPP, carbon export, phytoplankton composition, and stoichiometry. We ask the following questions: (1) Does surface ocean warming covary inversely with nutrient inventories? (2) Do planktonic communities display a stable or variable taxonomic and elemental composition? and, (3) How do these interactions impact observed carbon export?

\section{Results and discussion}

Trends in hydrography. Surface ocean temperatures significantly increased by $\sim 0.9^{\circ} \mathrm{C}$ over the entire duration of the BATS record analyzed here (1990-2020); however, the increase has not been uniform over time ${ }^{19,20}$. The 1990 s were characterized by a weak but significant increase in temperature at $10 \mathrm{~m}\left(R^{2}=0.01, P=0.007\right.$, $0.035 \pm 0.013{ }^{\circ} \mathrm{C}$ year $^{-1}$ ), whereas the following decade (2000s) did not exhibit a significant temperature trend $\left(R^{2}=0.00, P=0.493\right)$. The increase in near-surface temperature during the 2010s was fourfold greater than the $1990 \mathrm{~s}\left(R^{2}=0.10, P<0.001,0.119 \pm 0.008^{\circ} \mathrm{C}\right.$ year $^{-1}$ ) and accounted for the majority of the total temperature increase observed from 1990 to 2020 (Fig. 1A). This pattern of accelerated warming rates in the most recent decade has been previously shown ${ }^{20}$. Furthermore, D'Alelio et al. ${ }^{19}$, while examining the

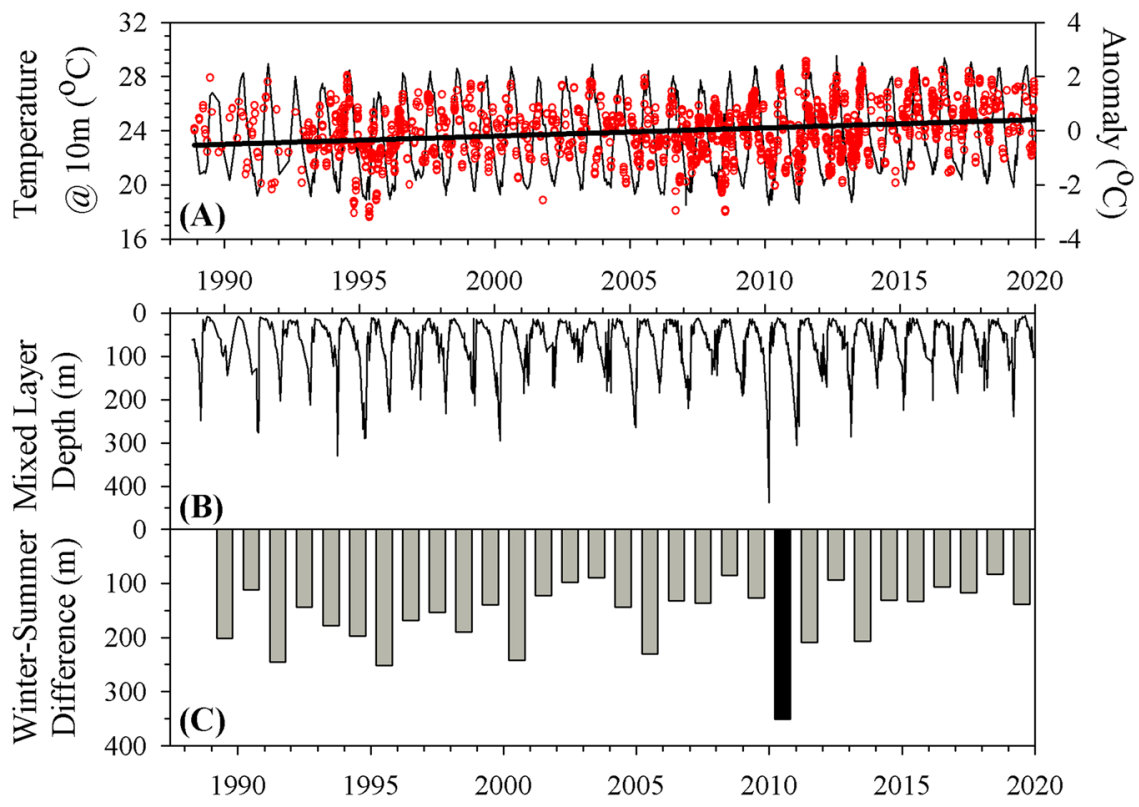

Fig. 1 Time series of surface ocean temperature (@10 $\mathbf{m}$ ), mixed layer depth, and seasonal mixing amplitude. A CTD temperature recorded at $10 \pm 2 \mathrm{~m}$ (thin black line), and seasonally detrended anomaly (red circles). The thick line is the Model 1 linear regression to the anomaly data. B Mixed layer depths estimated from each CTD cast in the BATS record using the $0.2^{\circ} \mathrm{C}$ variable sigma $-\theta$ criterion ${ }^{65}$. C Difference between average maximum winter/spring (February/March) and minimum summer (July/August) mixed layer depths for each year. Note: 2010 is highlighted in a different color for reasons discussed in the text. 
BATS dataset through 2015, also showed a significant decade-scale shift from no significant temperature change to significant warming beginning in $\sim 2008$, consistent with our findings. The goal of this current analysis is not to determine precisely when the period of rapid warming began, but rather to compare the biogeochemical and ecological responses during this period of rapid warming (2010s) with a period of weak to no warming (1990-2000s).

This pattern of rapidly increasing temperature through the 2010s has been shown to increase the stability of the upper ocean and reduce the formation of nutrient-rich subtropical mode water that lies below the euphotic zone ${ }^{21}$, which, ultimately, negatively impacts subsequent vertical nutrient inputs. During the 2010s, but not prior decades, the difference between winter maximum and summer minimum mixed layer depths significantly decreased (Fig. $1 \mathrm{~B}, \mathrm{C} ; R^{2}=0.46, P=0.03$ ) due primarily to shallowing of the winter maximum. This pattern is consistent with the temporal pattern in the magnitude of the wintertime North Atlantic Oscillation (NAO) index (Supplementary Fig. 1), which correlates with a range of hydrographic, chemical, and biological parameters at $\mathrm{BATS}^{22,23}$, and is inversely related to the depth of water column mixing during the winter period.

The increase in temperature and decrease in the amplitude of seasonal mixing through the 2010s was correlated with a significant net decline in inventories $(0-140 \mathrm{~m})$ of nitrate (Model 1 Regression, $P<0.001, R^{2}=0.34$, slope $=-11.6 \pm 1.6 \mathrm{mmol} \mathrm{m}^{-2}$ year $^{-1}$ ) and phosphate (Model 1 Regression, $P<0.001, R^{2}=0.36$, slope $=-0.43 \pm 0.06 \mathrm{mmol} \mathrm{m}^{-2}$ year $^{-1}$ ) (Fig. 2A, B). By the middle of the 2010 decade, nutrient inventories were reduced by $25-30 \%$ relative to their pre-2010 inventories (during which time there were no significant changes). The high-sensitivity phosphate record only goes back to 2004, but the standard autoanalyzer phosphate measurements collected at BATS also show no significant trend in inventories between 1990 and 2010 (see ref. ${ }^{24}$ ). It is noteworthy that the anomalous 2010 wintertime $\mathrm{NAO}$ index ${ }^{25}$ resulted in a significant increase in nutrient inventories from which this decade-scale decline began. Throughout the 2010s, the dissolved organic phosphorus (DOP) inventory also decreased significantly (Supplementary Information and
Supplementary Fig. 2). As predicted by global models ${ }^{5}$, the observed increase in surface temperature and reduction in nutrient inventories throughout the 2010s are correlated with a decade-long significant reduction in net primary production ( 30\%; NPP; Fig. 2C; Model 1 regression, $P=0.002, R^{2}=0.09$, slope $=-22.2 \pm 6.8 \mathrm{mg} \mathrm{m}^{-2}$ year $^{-1}$ ).

Trends in carbon fluxes, phytoplankton community and elemental stoichiometry. While global biogeochemical models predict this coordinated series of processes-ocean warming, increasing stratification, reduction in nutrient inputs, and subsequent decrease in NPP-they also predict a decrease in carbon export, thus reducing the magnitude of the biological carbon pump ${ }^{26,27}$. However, at BATS, counter to predictions, carbon export fluxes at $150 \mathrm{~m}$ did not significantly decrease throughout the 2010s (Model 1 Regression, $P=0.785$ ) (Fig. 3A, B). While it is interesting that the increased nutrient inventory and NPP in 2010, associated with the anomalous winter NAO negative index, did not result in a significant increase in carbon export, there are several possible explanations for decoupling of carbon export and NPP in response to episodic events ${ }^{28}$. Despite decreasing nutrient inventories and NPP, the lack of significant decrease in carbon export suggests that these ecosystem processes became decoupled throughout the 2010s. Furthermore, the export ratio (carbon export:NPP) increased from 0.04 in 2010 to 0.16 in 2019, indicating that the ecosystem became more efficient at exporting carbon from the euphotic zone, despite the decade-long decrease in nutrient inventories and NPP.

The decline in NPP throughout the 2010s was concurrent with a reorganization of the phytoplankton community (Fig. 4). Throughout the 2010s, the distinct seasonal peak in eukaryotic biomass diminished, and their biomass remained at a baseline level statistically similar to minimum values before 2010 (Fig. 4B). Throughout the 2010s, there were no contemporaneous changes in either seasonal biomass peaks or concentrations for prokaryotic Prochlorococcus and Synechococcus biomass (Fig. 4A). The decrease in eukaryote biomass resulted in a significant decrease in summed phytoplankton biomass throughout the 2010 decade
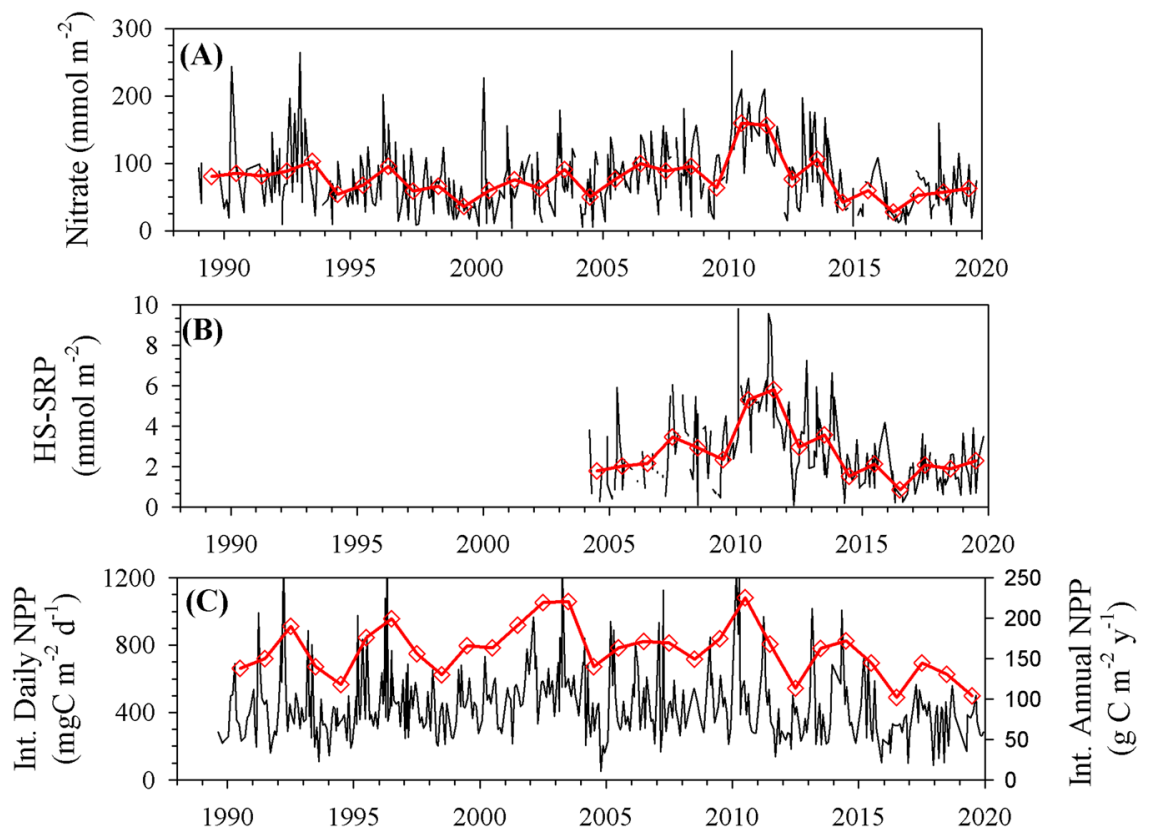

Fig. 2 Time series of surface ocean macronutrient inventories and integrated daily and annual net primary production (NPP). A Nitrate and B highsensitivity phosphate nutrient inventories $\left(0-140 \mathrm{~m} ; \mathrm{mmol} \mathrm{m}^{-2}\right)$, and $\mathbf{C}$ integrated $(0-140 \mathrm{~m})$ daily and annual NPP. Black lines in each panel are individual cruise values, while the red lines and open diamonds are annual values. Note, annual NPP is on a different scale (right axis). 


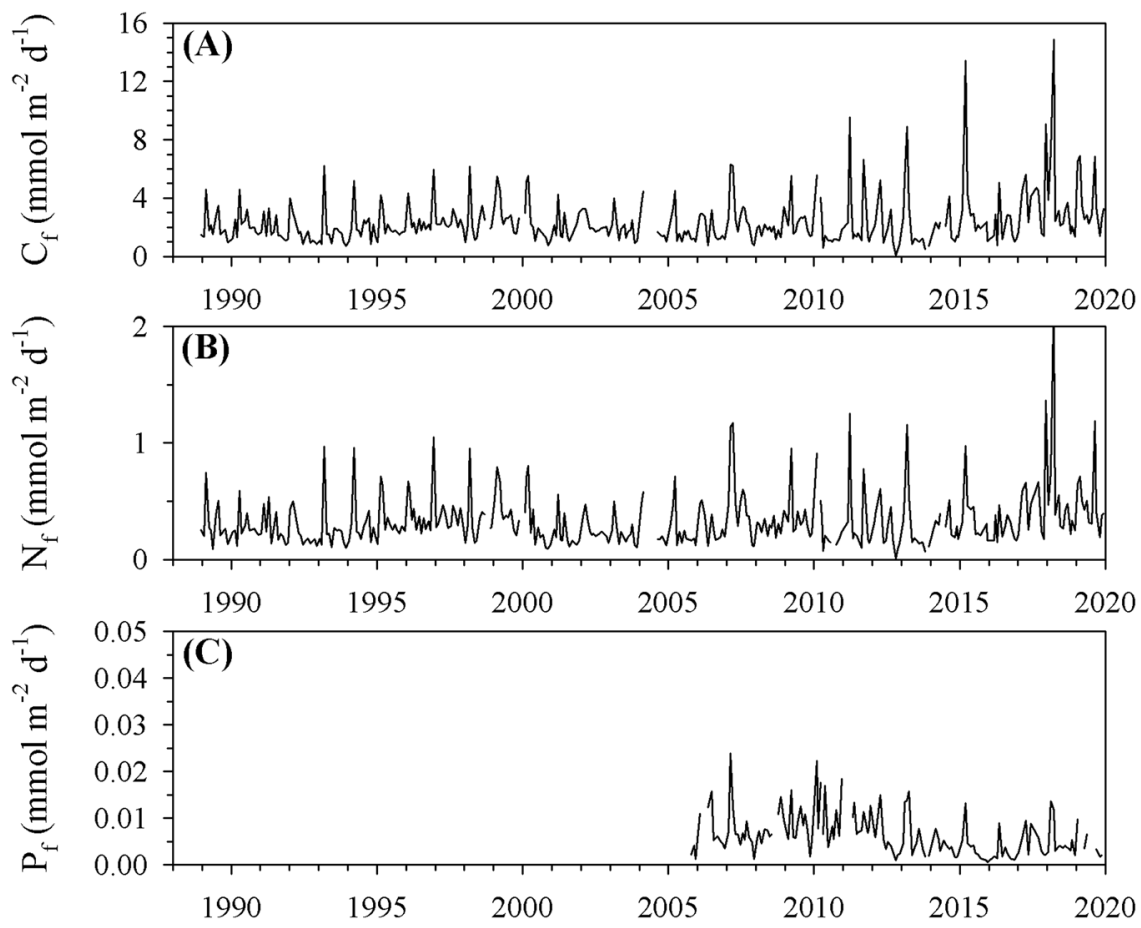

Fig. 3 Time series of vertical elemental fluxes at $\mathbf{1 5 0} \mathbf{m}$. A Particulate organic carbon $\left(C_{f}\right)$ flux; B particulate organic nitrogen $\left(N_{f}\right)$ flux; $\mathbf{C}$ particulate phosphorus $\left(P_{f}\right)$ flux.

(FCM PhytoC, $P<0.001$, slope $=-0.08 \pm 0.2 \mathrm{~g} \mathrm{C} \mathrm{m}^{-2}$ year $^{-1}$; Total PhytoC, $P<0.001$, slope $=-0.05 \pm 0.01 \mathrm{~g} \mathrm{C} \mathrm{m}^{-2}$ year $^{-1}$ ). We were unable to detect changes in the biomass of larger, less abundant microphytoplankton, as the summed prokaryote and small eukaryote carbon biomass estimates by FCM were only marginally greater than the independently estimated total phytoplankton carbon (ANOVA, FCM PhytoC vs. Total PhytoC pre-2010, $P=0.08$; FCM PhytoC vs. Total PhytoC post-2010, $P=0.04$ ) (Fig. 4C). In addition to changes in phytoplankton biomass, we examined changes in phytoplankton growth rate. Phytoplankton growth rates, derived by separately dividing integrated NPP by FCM PhytoC and Total PhytoC, did not show significant trends, increases or decreases, in any decade (Model 1 regression, $P>0.2$ in all comparisons) (Fig. 4D). Furthermore, mean growth rates pre- and post-2010 were not significantly different (Student's $t$ test, $P>0.4$ ) from each other. Thus, the $\sim 40 \%$ decline in NPP mentioned previously appears due primarily to the reduction in phytoplankton biomass during the 2010s, not a change in physiological condition. This change in the relative abundance of phytoplankton populations, and subsequent reduction in effective mean cell size of the phytoplankton population with the decrease of larger eukaryotes, only further confounds the decoupling of nutrients, NPP, and carbon export fluxes. Thus, explanations for the decoupling of nutrients, NPP, and carbon export likely involve changes in both phytoplankton processes (e.g., variable stoichiometry) and ecosystem processes (e.g., trophic interactions).

Decoupling of carbon and nutrient cycles and mechanisms to maintain carbon export. The Sargasso Sea is one of several western ocean gyre regions characterized by extremely low phosphate concentrations ${ }^{29}$, and is generally viewed as a phosphorus-limited system ${ }^{30-32}$. As such, changes in C:P stoichiometry are commonly studied in this region. Flexible macronutrient stoichiometry, associated with changes in phytoplankton community composition, has been hypothesized to buffer reductions in carbon export under conditions of increased stratification and nutrient depletion ${ }^{14}$. Based upon Tanioka and Matsumoto's sensitivity ratio ${ }^{14}$ and the observed decrease in phosphate inventory of $\sim 25-30 \%$, we would expect a reduction in carbon export of $\sim 18-22 \%$. Throughout the 2010s, when nutrient inventories and NPP were decreasing, there were significant and concurrent changes in the macronutrient stoichiometric ratios of exported material (Fig. 5). Declining phosphorus export fluxes throughout the 2010s (Fig. 3C), without decreases in carbon and nitrogen fluxes, resulted in a significant increasing trend in C:P (Model 1 regression, $P<0.001$, slope $=86 \pm 13$ units year $^{-1}$ ) and $\mathrm{N}: \mathrm{P}$ ratios (Model 1 regression, $P<0.001$, slope $=11.4 \pm 1.8$ units year $\left.^{-1}\right)$. This trend in increasing $\mathrm{C}: \mathrm{P}$ and $\mathrm{N}: \mathrm{P}$ ratios led to a nearly fourfold increase in the C:P $(205 \pm 108$ versus $722 \pm 226)$ and N:P $(29 \pm 14$ versus $101 \pm 35)$ stoichiometric ratios of exported material by the end of the 2010s when compared to the pre-2010 period (Fig. 5B, C), both of which were significant (Student's $t$ test, $P<0.001$ for both comparisons). A nutrient inversion model yielded a similar C:P ratio of exported material $(355 \pm 65)$ in the subtropical North Atlantic Ocean ${ }^{33}$ but did not resolve temporal changes in the ratio. As both carbon and nitrogen export flux rates did not decrease, the C:N stoichiometric ratio in exported material at $150 \mathrm{~m}$ depth did not significantly change between the period before and after 2010 and averaged $7.23 \pm 1.61$ (Fig. 5A). This temporal pattern in export flux rates and all stoichiometric ratios was also observed at the $200 \mathrm{~m}$ and $300 \mathrm{~m}$ sediment trap depths (Supplementary Figs. 2 and 3). However, the change in absolute rates decreased with depth due to organic matter remineralization ${ }^{34}$. The increases in $\mathrm{C}: \mathrm{P}$ and $\mathrm{N}: \mathrm{P}$ stoichiometric ratios of exported particulate material could be due to changes in the stoichiometry of the source material (i.e., suspended particulate matter, in particular, phytoplankton), and/or due to enhanced shallow remineralization of $\mathrm{P}$ between the base of the euphotic zone $(100 \mathrm{~m})$ and the shallow sediment traps $(150 \mathrm{~m})$, resulting in nutrient trapping ${ }^{35}$. This nutrient trapping effect is not $100 \%$ efficient, as the integrated nutrient inventories do continue to decline. However, we hypothesize that this effect slows down the rate of nutrient inventory 

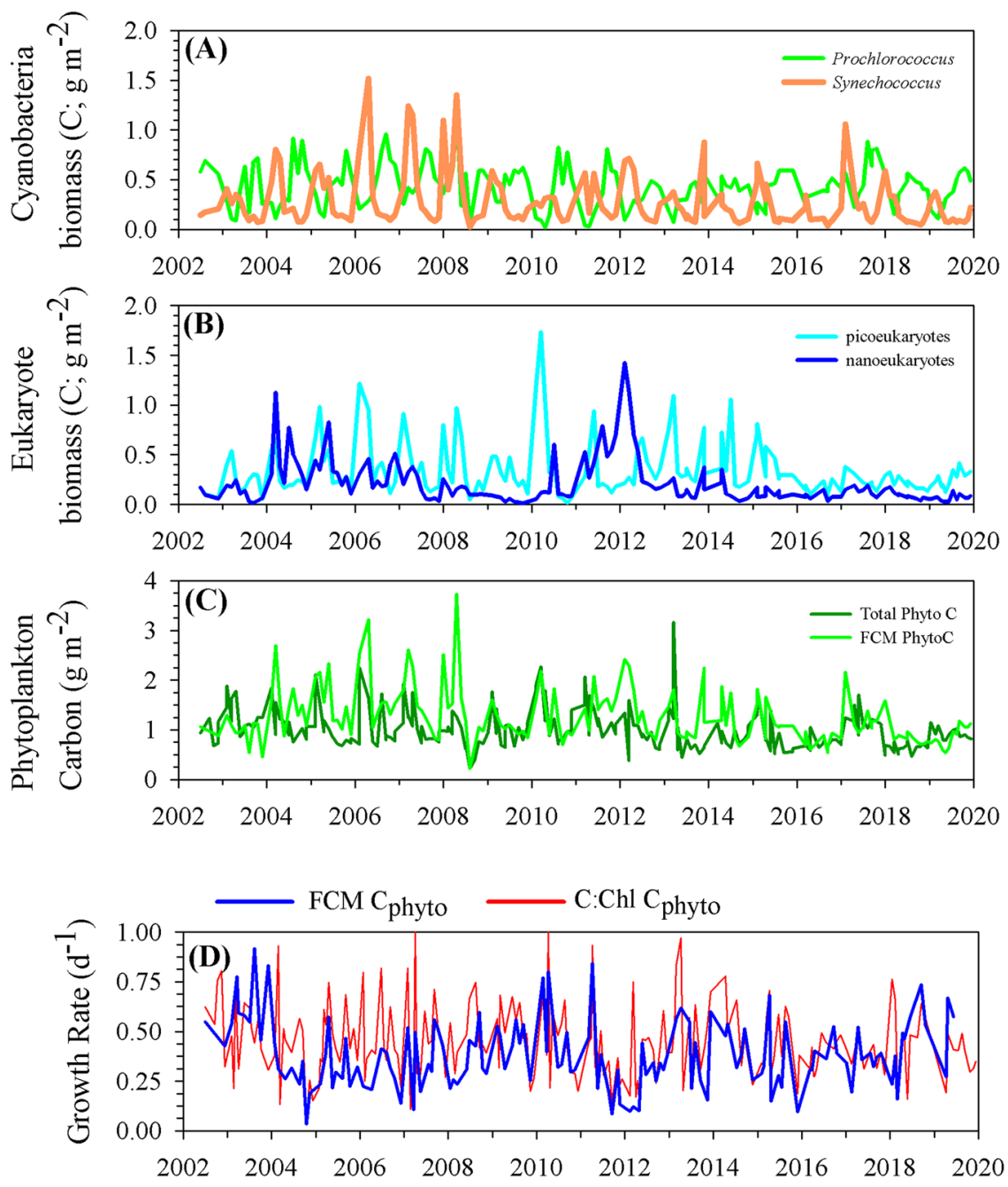

Fig. 4 Time series of flow cytometry-derived, euphotic zone integrated phytoplankton carbon biomass ( $\mathrm{g} \mathrm{m}^{-2}$ ) and phytoplankton growth rates.

A Biomass of cyanobacteria Prochlorococcus and Synechococcus; B biomass of picoeukaryotes and nanoeukaryotes; C total phytoplankton carbon biomass derived from the product of bulk POC:Chl slope and integrated Chl, and summed flow cytometry-derived carbon biomass estimates. D Phytoplankton growth rates estimated by dividing integrated in situ primary production by the two estimates of phytoplankton carbon biomass as described in (C).

decline and thus serves as another "biological buffer" to the negative impacts of upper-ocean stratification associated with climate change.

Stoichiometric ratios of sestonic particulate matter in the upper euphotic zone $(0-60 \mathrm{~m})$ displayed temporal patterns qualitatively similar to the same ratios in exported flux ratios (Fig. 6). C:P ratios in suspended particulate organic matter increased significantly (Model 1 Regression, $R^{2}=0.17, P<0.001$, slope $=14.5 \pm 2.9$ units year $^{-1}$ ) throughout the 2010s and reached values higher than in the 2000s. The N:P ratio, constrained by both elements potentially being limited, also increased significantly (Model 1 Regression, $R^{2}=0.08, P=0.02$, slope $=1.48 \pm 0.5$ units year $\left.^{-1}\right)$. Stoichiometric ratios in the lower euphotic zone (60-100 m; Supplementary Fig. 4) also mirrored temporal patterns in the upper euphotic zone but were lower in magnitude. C:P ratios in the lower euphotic zone were $~ 17 \%$ lower than in the upper euphotic zone, while N:P ratios were $\sim 30 \%$ less, supporting the notion that the lower euphotic zone may be less nutrient stressed due to proximity to diapycnal nutrient inputs that are efficiently assimilated ${ }^{36}$. The seston pool comprises many particle types, including heterotrophic microbes, phytoplankton, microzooplankton, and detrital particles. Phytoplankton comprise a significant fraction of the total POC pool, ranging from 0.11 to $0.71(0.30 \pm 0.11$; Supplementary Fig. 5). Thus, we posit that at least a portion of the increase in seston C:P ratios is linked to the shift towards increasing dominance of the phytoplankton community by cyanobacteria (Fig. 4), which are well documented to have C:P ratios in natural populations significantly greater than co-occurring eukaryotes ${ }^{15,16,37}$.

While data on long-term changes in the phytoplankton community exists, we do not have comparable time series of phytoplankton elemental stoichiometry. Using a trait-based phytoplankton model coupled with satellite inputs ${ }^{38}$, we estimated long-term patterns in the phytoplankton C:P ratio in the Sargasso Sea (Fig. 7). Over the available temporal record, 2003-2020, the modeled phytoplankton C:P ratio was similar in magnitude to the bulk seston C:P ratio (Fig. 6). The modeled phytoplankton C:P ratio significantly increased throughout the 2010s (Model 1 Regression, $P=0.002$; slope $=4.3 \pm 1.4$ units year $\left.{ }^{-1}\right)$, which due to high variance, is statistically similar to the increase in the seston C:P ratio $(P<0.001$; slope $=14.5 \pm 2.9$ units year $\left.{ }^{-1}\right)$. The change in modeled phytoplankton C:P is based upon using Synechococcus to represent the phytoplankton community, due to lack of validation datasets for other phytoplankton taxa, but this should also qualitatively reflect the change in the C:P Prochlorococcus ${ }^{39}$, which combined, constitute the largest portion of phytoplankton in the Sargasso Sea ${ }^{40}$. This increase in modeled phytoplankton C:P, for a taxonomically 


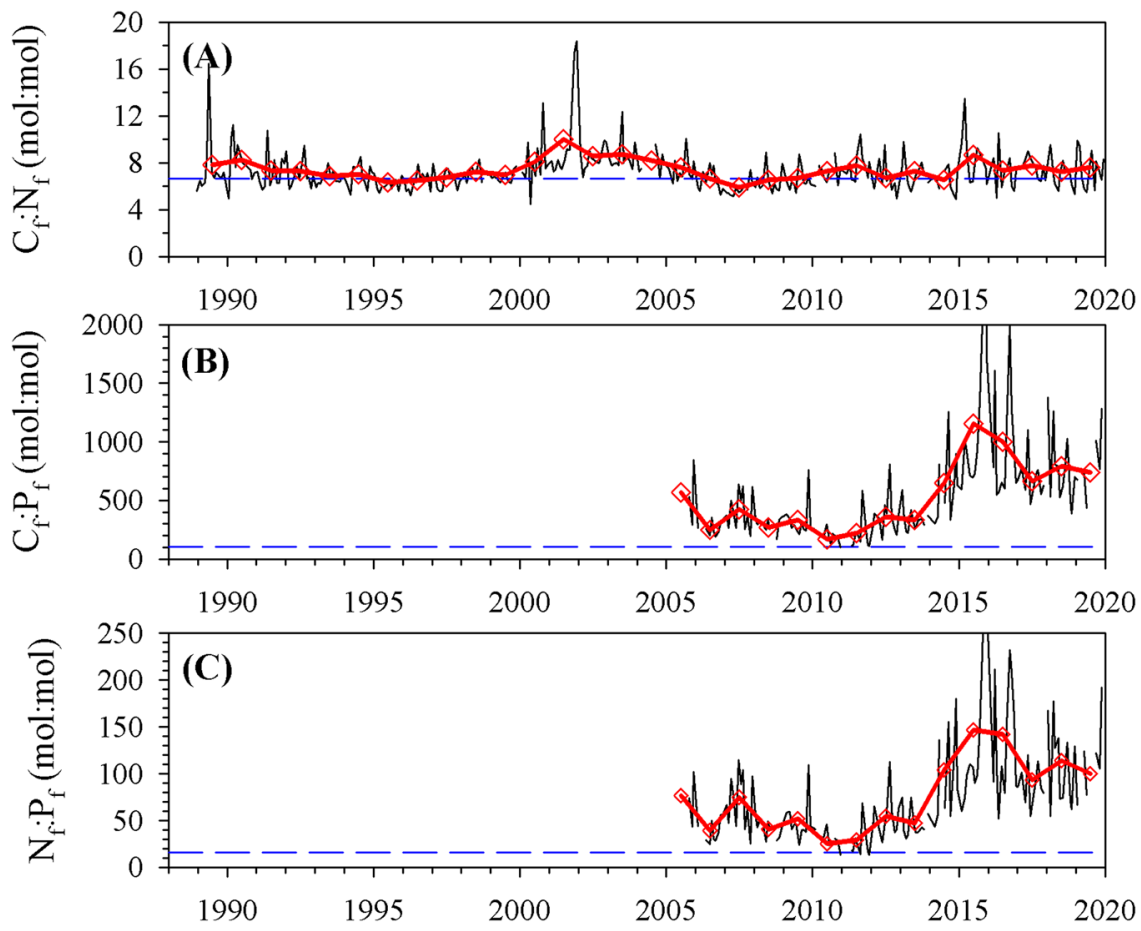

Fig. 5 Time series of elemental flux stoichiometric ratios at $\mathbf{1 5 0} \mathbf{~ m}$. A Particulate $C_{f}: N_{f}$ flux ratio (thin black line); B particulate $C_{f}: P_{f}$ flux ratio; and C particulate $N_{f}: P_{f}$ flux ratio. Red triangles represent the annual mean flux ratio, and the blue dashed line represents the Redfield Ratio.
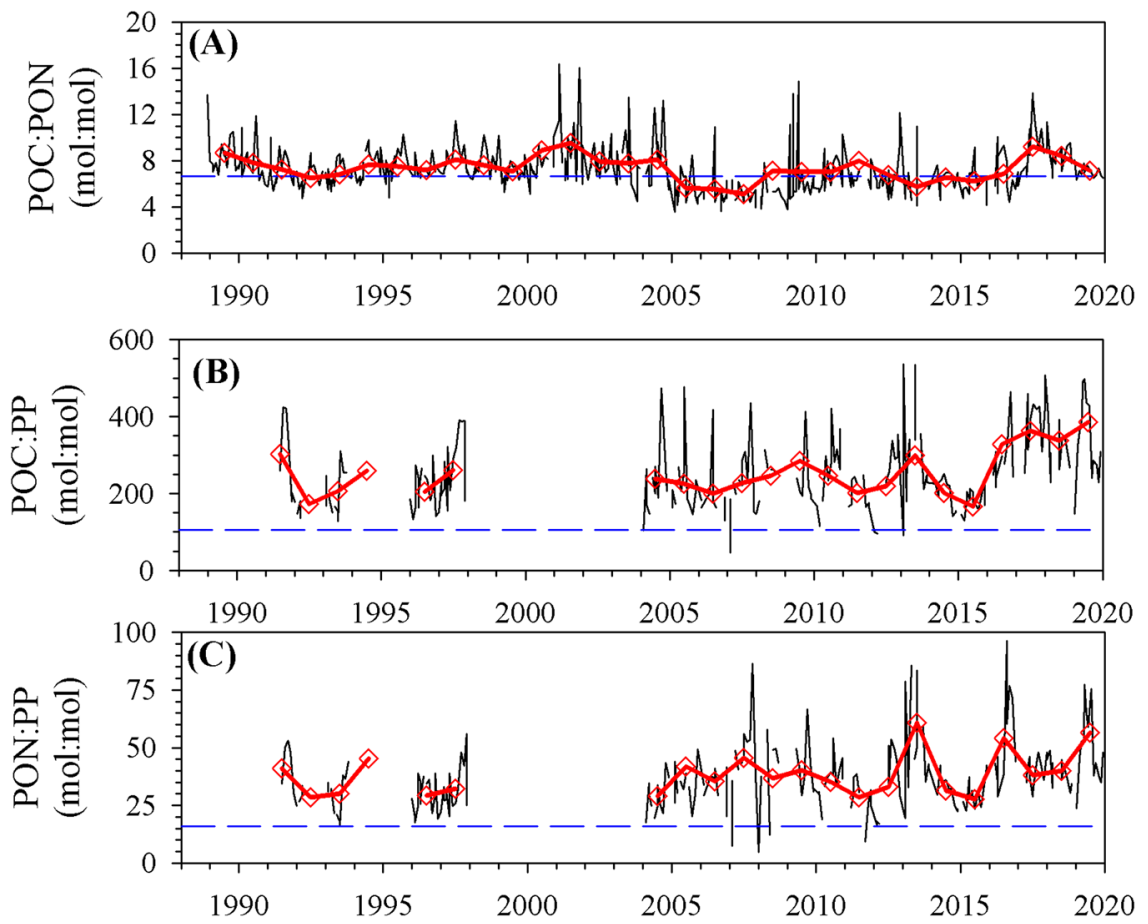

Fig. 6 Time series of seston elemental stoichiometric ratios in the upper (0-60 m) euphotic zone layer. A POC:PON; B POC:PP; C PON:PP. Red triangles represent the annual mean stoichiometric ratio, and the blue dashed line represents the Redfield Ratio.

"constant" phytoplankton assemblage, is hypothesized to be due to slight decreases in growth rates that have a disproportionate impact on C:P ratios ${ }^{38}$. Neither the observational estimates of phytoplankton growth rate based upon carbon nor the modeled growth rate (Supplementary Fig. 9) significantly decreased through the 2010s, but all displayed a negative slope (Fig. 4D). This suggests that physiological adjustments that increased cellular C:P ratios had not yet resulted in significant decreases in growth rate.

While the temporal patterns of stoichiometric ratios in the euphotic zone mirrored those of exported particulate matter, C:P ratios in the euphotic zone were consistently and significantly lower (sample-to-sample Student's $t$ test; $P<0.001$ ) than ratios at the $150-\mathrm{m}$ sediment trap, suggesting enhanced shallow 


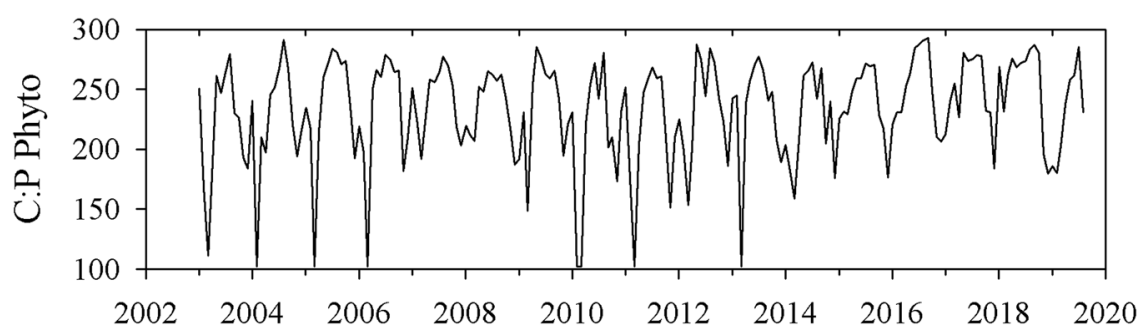

Fig. 7 Time series of phytoplankton-specific C:P ratios calculated from the trait-based model approach of Tanioka et al. ${ }^{38}$. Model phytoplankton C:P in the surface $100 \mathrm{~m}$ is computed as a function of growth rate and POC:Chl-a ratio derived from MODIS-Aqua combined with a map of temperaturedependent nutrient limitation.

remineralization of both $\mathrm{P}$ and $\mathrm{N}$ between 100 and $150 \mathrm{~m}^{41,42}$. Most clearly, for the coupling of C:P ratios, it appears that both changes in the ratio of source material and enhanced remineralization of $\mathrm{P}$ are important. Based on the change over time in seston C:P ratios relative to changes over time in C:P ratios of exported material, we estimate $\sim 20 \%$ of the change in the ratio of exported material is due to this change in the source material. By difference, $\sim 80 \%$ of the change is due to enhanced shallow demineralization, which has been hypothesized as an important mechanism to sustain carbon export in oligotrophic regions ${ }^{43}$. Data for bacterial productivity between 100 and $150 \mathrm{~m}$ is scant during the decade of the 2010s, thus we cannot assess if the apparent increase in shallow remineralization is associated with changes in bacterial productivity, zooplankton "repackaging" 44 , and/or other unidentified processes.

Pico- and nanoeukaryotes in the Sargasso Sea are estimated to contribute $\sim 10 \%$ of carbon export, which is at or below their contribution to carbon biomass-however, the precise mechanisms are poorly known ${ }^{17}$. Thus, while the loss of small eukaryotes should have had a detrimental impact on carbon export, it is conceivable that the resulting increase in the relative contribution of cyanobacteria with elevated C:P ratios maintained carbon export rates. Laboratory ${ }^{45,46}$ and field ${ }^{47-49}$ studies in the Sargasso Sea have confirmed that cyanobacteria are incorporated into large phytodetrital aggregates and fecal aggregates through physical interaction and "sticking", thus potentially increasing their importance in carbon export. Phytodetrital aggregates alone have been shown to contribute $30-70 \%$ of measured bulk POC flux ${ }^{47}$. In contrast, studies have clearly shown that these picoplankton populations are tightly controlled by micrograzers ${ }^{50-52}$ and that "excess" carbon, relative to the needs of microzooplankton grazers, is respired during grazing ${ }^{53}$. Thus, this tight micrograzer control could counter an increased contribution of these picophytoplankton to carbon export.

Another mechanism for the lack of reduction in carbon export is an extensive but changing role of macrograzers as mediators of carbon flux; a hypothesis consistent with the high frequency of "zooplankton gut microbes" associated with fecal aggregates ${ }^{47}$. Salps are gelatinous filter feeders that can also effectively feed on picophytoplankton ${ }^{54}$ and they produce large, fast-sinking fecal pellets $^{55}$. In contrast to crustacean zooplankton and microzooplankton, salps do not appear to respire excess carbon from their food leading to C:P ratios in their fecal material equal to or greater than suspended POM and the C:P ratio in exported material (Supplementary Fig. 8). Salp blooms occur periodically in the Sargasso Sea, dominating the zooplankton community and carbon export. An analysis of salp abundance at BATS from 1994 to 2011 indicates a long-term increase in the most common salp species $^{56,57}$, which is positively correlated with a water column stratification index (analogous to our seasonal mixing depth amplitude). While the time series post-2011 has not been analyzed specifically for salps, we examined a metric that could reflect changes in salp abundance, zooplankton dry to wet weight ratio (in which an increase in salp abundance would result in a relatively lower ratio), however, no significant trends were observed. We also examined size-fractioned zooplankton biomass data to determine more broadly if there have been changes in community structure. Both day- and nighttime absolute zooplankton biomass of the largest $(>5 \mathrm{~mm})$ size class increased significantly in the period before 2010 , and significantly decreased in the 2010s decade (Model 1 Regression, LN normalized data, $P<0.001$ ) (only nighttime data shown, Supplementary Fig. 7a), however the fraction of zooplankton biomass $>5 \mathrm{~mm}$ relative to total zooplankton biomass $>0.2 \mathrm{~mm}$ did not change significantly before and after 2010 (Supplementary Fig. 7b), suggesting no change in the relative size structure of the zooplankton over the entire time series. At this time, we cannot sum these potential food web trophic changes with enough confidence to evaluate their contributions to maintaining elevated carbon export rates in the face of reduced phytoplankton biomass and mean population cell size.

We identify a counter-intuitive biogeochemical response as a result of warming in the subtropical Sargasso Sea. The 2010s saw warming faster than other decades in the past 30 years, depletion of euphotic zone nutrient inventories in response to more intense seasonal stratification, and the anticipated reduction in NPP. What was not observed is a coordinated reduction in carbon export, despite a relative increase in cyanobacteria and a decrease in total phytoplankton biomass, as the ecosystem improved its $\mathrm{P}$ use efficiency. These results suggest changes in the phytoplankton community and trophic interactions controlling the continued export of carbon and remineralization of limiting nutrients are not adequately captured in current earth system models.

\section{Methods}

Study location and overview. The history of the Sargasso Sea ocean time-series research and basic understanding of the physical and biological characteristics of this region are described in detail in prior reviews ${ }^{24,58,59}$. On each monthly (biweekly in the spring) BATS cruise, a quasi-lagrangian sampling scheme is employed, and for this manuscript, all CTD/hydro casts falling within a $0.25^{\circ}$ latitude by $0.25^{\circ}$ longitude box centered around the BATS site $\left(31^{\circ} 40^{\prime} \mathrm{N} ; 64^{\circ} 10^{\prime} \mathrm{W}\right)$ are presented. Further details of the sampling scheme, analytical methods, data quality control $(\mathrm{QC})$ and quality assurance $(\mathrm{QA})$, and history of sampling procedures are available in the BATS methods manuals ${ }^{60}$ and in published papers ${ }^{61-64}$. Detailed methods are provided below.

Hydrography. Continuous CTD data were collected from the downcast and calibrated against discrete samples (e.g., ref. ${ }^{24}$ ). CTD profile data were used to estimate mixed layer depths using a $0.2^{\circ} \mathrm{C}$ variable sigma- $\theta$ criterion $^{65}$. Near-surface $(10 \mathrm{~m})$ temperature anomalies were generated by seasonally detrending the entire data record.

Dissolved inorganic, organic, and particulate nutrients. Samples for nitrate and phosphate were filtered $(0.8-\mu \mathrm{m}$ polycarbonate filter) into acid-washed HDPE bottles, and frozen $\left(-20^{\circ} \mathrm{C}\right)$ until analysis using standard air-segmented autoanalyzer methods ${ }^{66}$. In addition, starting in October 2004, samples were collected for high-sensitivity phosphate analyses using the Magnesium Induced Coprecipitation (MAGIC) soluble reactive phosphorus (SRP) method ${ }^{67,68}$, as modified for BATS ${ }^{31}$. During each sample run, commercially available certified 
standards, OSIL and Wako Chemical, are analyzed to maintain consistent data quality, as well as "standard water" from $3000 \mathrm{~m}$ that serves as an internal standard.

Particulate organic carbon (POC), nitrogen (PON), and phosphorus (PP) samples were filtered on precombusted $\left(450^{\circ} \mathrm{C}, 4 \mathrm{~h}\right)$ Whatman $\mathrm{GF} / \mathrm{F}$ filters and frozen until analysis on a Control Equipment 440-XA elemental analyzer ${ }^{59}$. Particulate phosphorus samples (PP) were analyzed using an ash-hydrolysis method with oxidation efficiency and standard recovery checks ${ }^{31}$.

Picoplankton abundance and biomass. Samples for pico- and nanoplankton enumeration were collected on each cruise from June 2002 to the present ${ }^{69}$. Samples were preserved $(0.5 \% \mathrm{v} / \mathrm{v}$ paraformaldehyde) and flash-frozen in liquid nitrogen before being stored at $-80^{\circ} \mathrm{C}$ until analysis by flow cytometry. Small cyanobacteria were identified as either Synechococcus or Prochlorococcus based upon cell size and the presence or absence of phycoerythrin, respectively. Eukaryotes were defined as other chlorophyll-containing cells not being cyanobacteria. They were separated into picoeukaryotes $(<3 \mu \mathrm{m})$ and nanoeukaryotes $(>3 \mu \mathrm{m})$ based upon their forward scatter signal relative to $3-\mu \mathrm{m}$ polystyrene beads. Phytoplankton cell abundance was converted to carbon per cell using a normalized cell size-carbon relationship and then to population biomass by multiplying by cell abundance $\left({ }^{40}\right)$

Total phytoplankton carbon was estimated independently by regressing integrated $(0-140 \mathrm{~m})$ total POC against total chlorophyll $(\mathrm{Chl}-a)$ to obtain a single slope for the dataset that relates the two parameters. This approach was used rather than taking the average ratio of discrete POC and Chl- $a$ integrals as it allows for the exclusion of "non-phytoplankton" POC (i.e., the $y$ intercept in the regression) from the relationship between phytoplankton POC and Chl-a. The slope of the POC:Chl- $a$ regression was then multiplied by integrated Chl- $a$ to estimate phytoplankton POC. This estimate of total phytoplankton carbon was compared to the sum of flow cytometry-derived picoplankton carbon, with any differences indicative of larger microplankton not well measured by flow cytometry.

Particulate elemental fluxes. Sinking fluxes of POC, PON, PP from the euphotic zone were quantified using surface-tethered particle interceptor traps ${ }^{24,59,70}$. Fluxes were calculated from the elemental mass of material captured in the sediment trap, scaled to the sediment trap tube surface area and array deployment duration. A filter blank correction, based on three blank particle interceptor traps, was applied to POC and PON fluxes starting in October 2008. Paired analysis of "raw" and "blank-corrected" flux rates show that the blank correction was $2.3 \%$ of raw POC fluxes and $4.5 \%$ of PON fluxes across the entire range of fluxes. These blank corrections were applied uniformly to the entire data record to provide consistency throughout the whole data record.

Primary production. In situ production estimates were determined using surfacetethered arrays with samples spaced every $20 \mathrm{~m}$ between the surface and $140 \mathrm{~m}$. Rates of primary production were calculated from the incorporation of $\mathrm{H}^{14} \mathrm{CO}_{3}$ into particles (i.e., particles nominally $>0.7 \mu \mathrm{m}$ ) using an assumed ratio of total inorganic carbon present to radiocarbon added. From 1990 through 2005, samples were collected with Go-Flo bottles on a Kevlar line, and from November 2004 to the present, samples were collected from Niskin bottles on the CTD rosette. Rates of primary production were corrected for dark carbon uptake and integrated to a depth of $140 \mathrm{~m}^{23}$.

Trait-based model. The trait-based phytoplankton stoichiometry model is a modification of Inomura et al..$^{71}$, which facilitates the accurate computation of phytoplankton C:N:P under a variety of environmental conditions. Here, the original trait-based based model was modified following Tanioka et al. ${ }^{38}$ to predict phytoplankton C:P as a function of satellite-based growth rate, Chl:C, and $\mathrm{P}$ limitation. We used the model parameter set for the freshwater cyanobacteria Synechococcus linearis except for a hard-bound maximum C:P of 335 at the zero growth rate, following experimental chemostat data on the marine cyanobacteria Synechococcus WH8102 $2^{72}$. With this slight modification to the model parameters, the model C:P matches the observed C:P from the culture experiment when the growth rate is less than $0.8 \mathrm{~d}^{-1}$ (Supplementary Fig. 7). The model overestimates the observed C:P by $\sim 40$ at the highest growth rate, possibly due to the simplified nature of the storage pool representation. However, as the typical seasonal maximum growth rate observed at BATS rarely exceeds 0.8 day $^{-1}$ (Fig. 4C), the model can accurately predict satellite-driven C:P.

We report here a monthly and area-averaged C:P for a 3-by-3 pixel around BATS predicted using the trait-based model forced with satellite input derived from MODIS-aqua. Satellite-derived monthly growth rate and phytoplankton carbon $\left(\mathrm{C}_{\text {phyto }}\right)$ are based on the estimates from the Carbon-based Productivity Model $\left(\mathrm{C}_{\mathrm{bPM}}\right)^{73}$. Chl:Cphyto ratio, a proxy for light limitation, is computed by dividing MODIS-derived Chl- $a$ with $\mathrm{C}_{\text {phyto }}$. Both growth rate and $\mathrm{Chl}_{\mathrm{C}} \mathrm{C}_{\text {phyto }}$ are assumed to be vertically uniform in the mixed layer. $P$ limitation is inferred by comparing MODIS-derived monthly mean SST and empirically derived phosphate depletion temperature, a temperature above which phosphate is no longer detectable ${ }^{74}$.

\section{Data availability}

All observational data for the BATS time series can be found either on the BATS data server (http://bats.bios.edu/bats-data/) or on the BATS project page at the BiologicalChemical Oceanography Data Management Office (https://www.bco-dmo.org/project/ 2124). Output from the trait-based model, and its code, can be reasonably requested directly from co-author Tatsuro Tanioka.

Received: 3 July 2021; Accepted: 7 February 2022; Published online: 08 March 2022

\section{References}

1. Falkowski, P. G., Barber, R. T. \& Smetacek, V. Biogeochemical controls and feedbacks on ocean primary productivity. Science 281, 200-206 (1998).

2. Harris, G. Phytoplankton Ecology: Structure, Function and Flucuation (Springer Science and Business Media, 2012).

3. Boyce, D., Lewis, M. \& Worm, B. Global phytoplankton decline over the past century. Nature 466, 591-596 (2010).

4. Martinez, E., Antoine, D., D’Ortenzio, F. \& Gentili, B. Climate-driven basinscale decadal oscillations of oceanic phytoplankton. Science 326, 1253-1256 (2009).

5. Bopp, L. et al. Multiple stressors of ocean ecosystems in the 21st century: projections with CMIP5 models. Biogeosciences 10, 6225-6245 (2013).

6. Henson, S. A., Beaulieu, C. \& Lampitt, R. Observing climate change trends in ocean biogeochemistry: when and where. Glob. Change Biol. 22, 1561-1571 (2016).

7. Kwiatkowski, L., Aumont, O., Bopp, L. \& Ciais, P. The impact of variable phytoplankton stoichiometry on projections of primary production, food quality and carbon uptake in the global ocean. Glob. Biogeochem. Cycle 32, 516-528 (2018).

8. Toseland, S. et al. The impact of temperature on marine phytoplankton resource allocation and metabolism. Nat. Clim. Change 3, 979-984 (2013).

9. Skyllas, N. et al. Validation of stratification-driven phytoplanton biomass and nutrient concentrations in the northeast Atlantic Ocean as simulated by ECEarth. Geosciences 9, https://doi.org/10.3390/geosciences9100450 (2019).

10. Bopp, L., Aumont, O., Cadule, P., Alvain, S. \& Gehlen, M. Response of diatoms distribution to global warming and potential implications: A global model study. Geophys. Res. Lett. 32, L19606. 19610.11029/12005GL023653 (2005).

11. Doney, S. C. et al. Climate change impacts on marine ecosystems. Annu. Rev. Mar. Sci. 4, 11-37 (2012).

12. Redfield, A. On the Proportions of Organic Derivatives in Sea Water and Their Relation to the Composition of Plankton in James Johnstone Memorial Volume. (ed. R.J. Daniel) 176-192 (University Press of Liverpool, 1934).

13. Redfield, A. The biological control of chemical factors in the environment. Am. Scientist. 46, 205-221 (1958).

14. Tanioka, T. \& Matsumoto, K. Buffering of ocean export production by flexible elemental stoichiometry of particulate organic matter. Glob. Biogeochem. Cycle 31, 1528-1542 (2017).

15. Baer, S., Lomas, M., Terpis, K., Mouginot, C. \& Martiny, A. C. Stoichiometry of Prochloroccus, Synechococcus, and small eukaryotic populations in the western North Atlantic Ocean. Environ. Microbiol. 19, 1568-1583 (2017).

16. Martiny, A. C. et al. Strong latitudinal patterns in the elemental ratios of marine plankton and organic matter. Nat. Geosci. 6, 279-283 (2013).

17. Lomas, M. W. \& Moran, S. B. Evidence for aggregation and export of cyanobacteria and nano-eukaryotes from the Sargasso Sea euphotic zone. Biogeosciences 8, 203-216 (2011).

18. Brix, H., Gruber, N., Karl, D. \& Bates, N. Interannual variability of the relationship between primary, net community and export production in the subtropical gyres. Deep-Sea Res. II 53, 698-717 (2006).

19. D'Alelio, D. et al. Machine learning identifies a strong association between warming and reduced primary productivity in an oligotrophic ocean gyre. Sci. Rep. 10, https://doi.org/10.1038/s41598-41020-59989-y (2020).

20. Bates, N. \& Johnson, R. Acceleration of ocean warming, salinification, deoxygenation and acidification in the surface subtropical North Atlantic Ocean. Commun. Earth Envion. 1, https://doi.org/10.1038/s43247-4302000030-43245 (2020)

21. Stevens, S. W., Johnson, R. J., Maze, G. \& Bates, N. R. A recent decline in North Atlantic subtropical mode water formation. Nat. Clim. Change 10, 335-341 (2020).

22. Bates, N. R. Interannual variability of oceanic $\mathrm{CO}_{2}$ and biogeochemical properties in the Western North Atlantic subtropical gyre. Deep-Sea Res. Part II 48, 1507-1528 (2001).

23. Lomas, M. W. \& Bates, N. R. Potential controls on interannual partitioning of organic carbon during the winter/spring phytoplankton bloom at the Bermuda Atlantic Time-series Study (BATS) site. Deep-Sea Res. Part I 51, $1619-1636$ (2004). 
24. Lomas, M. W. et al. Two decades and counting: 24-years of sustained open ocean biogeochemical measurements in the Sargasso Sea. Deep Sea Res. II 93 , 16-32 (2013)

25. Osborn, T. J. Winter 2009/2010 temperatures and a record-breaking North Atlantic Oscillation index. Weather https://doi.org/10.1002/wea.1660 (2010).

26. Bopp, L. et al. Potential impact of climate change on marine export production. Glob. Biogeochem. Cycle 15, 81-99 (2001).

27. Fu, W., Randerson, J. T. \& Moore, J. K. Climate change impacts on net primary production (NPP) and export production (EP) regulated by increasing stratification and phytoplankton community structure in the CMIP5 models. Biogeosciences 13, 5151-5170 (2016).

28. Buesseler, $\mathrm{K}$. The decoupling of production and particulate export in the surface ocean. Glob. Biogeochem. Cycle 12, 297-310 (1998).

29. Martiny, A. C. et al. Biogeochemical controls of surface ocean phosphate. Sci. Adv. 5, eaax0341 (2019).

30. Ammerman, J. W., Hood, R. R., Case, D. \& Cotner, J. B. Phosphorus deficiency in the Atlantic: an emerging paradigm in oceanography. EOS 84, 165-170 (2003).

31. Lomas, M. W. et al. Sargasso Sea phosphorus biogeochemistry: an important role for dissolved organic phosphorus (DOP). Biogeosciences 7, 695-710 (2010).

32. Wu, J., Sunda, W., Boyle, E. \& Karl, D. Phosphate depletion in the western North Atlantic Ocean. Science 289, 759-762 (2000).

33. Teng, Y.-C., Primeau, F., Moore, J. K., Lomas, M. W. \& Martiny, A. C. Globalscale variations of the ratios of carbon to phosphorus in exported marine organic matter. Nat. Geosci. 7, 895-898 (2014).

34. Martin, J., Knauer, G. A., Karl, D. M. \& Broenkow, W. VERTEX: carbon cycling in the NE Pacific. Deep-Sea Res. Part a-Oceanographic Res. Pap. 34 267-285 (1987).

35. Buesseler, K. O. \& Boyd, P. W. Shedding light on processes that control particle export and flux attenuation in the twilight zone of the open ocean. Limnol. Oceanogr. 54, 1210-1232 (2009).

36. Cullen, J. \& Eppley, R. W. Chlorophyll maximum layers of the South California Bight and possible mechanisms of their formation and maintenance. Oceanol. Acta 4, 23-32 (1981).

37. Lomas, M. et al. Varying influence of phytoplankton biodiversity and stoichiometric plasticity on bulk particulate stoichiometry across ocean basins. Commun. Earth Envion. 2, 143 https://doi.org/10.1038/s43247-021-00212-9 (2021).

38. Tanioka, T., Fichot, C. \& Matsumoto, K. Toward determining the spatiotemporal variability of upper-ocean ecosystem stoichiometry from satellite remote sensing. Front. Mar. Sci. 7, 604893 (2020).

39. Bertilsson, S., Berglund, O., Karl, D. M. \& Chisholm, S. W. Elemental composition of marine Prochlorococcus and Synechococcus: implications for the ecological stoichiometry of the sea. Limnol. Oceanogr. 48, 1721-1731 (2003).

40. Casey, J., Aucan, J., Goldberg, S. \& Lomas, M. Changes in partitioning of carbon amongst photosynthetic pico- and nano-plankton groups in the Sargasso Sea in response to changes in the North Atlantic Oscillation. Deep Sea Res. II 93, 58-70 (2013).

41. Clark, L., Ingall, E. \& Benner, R. Marine phosphorus is selectively remineralized. Nature 393, 426 (1998).

42. Monteiro, F. \& Follows, M. On nitrogen fixation and preferential remineralization of phosphorus. Geophys. Res. Lett. 39, https://doi.org/ 10.1029/2012GL050897 (2012)

43. Tanioka, T., Matsumoto, K. \& Lomas, M. W. Drawdown of atmospheric $\mathrm{pCO}_{2}$ via dynamic particle export stoichiometry in the ocean twilight zone. Earth Space Sci. Open Arch. https://doi.org/10.1002/essoar.10507405.10507401 (2021).

44. Steinberg, D. et al. Bacterial vs. zooplankton control of sinking particle flux in the ocean's twilight zone. Limnol. Oceanogr. 53, 1327-1338 (2008).

45. Cruz, B. N. \& Neuer, S. Heterotrophic bacteria enhance the aggregation of the marine picocyanobacteria Prochlorococcus and Synechococcus. Front. Microbiol. https://doi.org/10.3389/fmicb.2019.01864 (2019).

46. Deng, W., Cruz, B. N. \& Neuer, S. Effects of nutrient limitation on cell growth, TEP production and aggregate formation of marine Synechococcus. Aquat. Microb. Ecol. 78, 39-49 (2016).

47. Cruz, B. N., Brozak, S. \& Neuer, S. Microscopy and DNA-based characterization of sinking particles at the Bermuda Atlantic Time-series Study (BATS) station point to zooplankton mediation of particle flux. Limnol. Oceanogr. https://doi.org/10.1002/lno.11910 (2021).

48. De Martini, F., Neuer, S., Hamill, D., Robidart, J. \& Lomas, M. W. Clade and strain specific contributions of Synechococcus and Prochlorococcus to carbon export in the Sargasso Sea. Limnol. Oceanogr. https://doi.org/10.1002/lno.10765 (2018).

49. Schnetzer, A. \& Steinberg, D. K. Natural diets of vertically migrating zooplankton in the Sargasso Sea. Mar. Biol. 141, 89-99 (2002).

50. Edwards, K. F. Mixotrophy in nanoflagellates across environmental gradients in the ocean. Proc. Acad. Nat. Sci. Phila. 116, 6211-6220 (2019).

51. Worden, A. Z. \& Binder, B. J. Application of dilution experiments for measuring growth and mortality rates among Prochlorococcus and Synechococcus populations in oligotrophic environments. Aquat. Microb. Ecol. 30, 159-174 (2003).
52. Zubkov, M. V. \& Tarran, G. A. High bacterivory by the smallest phytoplankton in the North Atlantic Ocean. Nature 455, 224-226 (2008).

53. Meunier, C., Haafke, J., Oppermann, B., Boersma, M. \& Malzahn, A. Dynamic stoichiometric response to food quality fluctuations in the heterotrophic dinoflagellate Oxyrrhis marina. Mar. Biol. 159, 2241-2248 (2012).

54. Dadon-Pilosof, A., Lombard, F., Genin, A., Sutherland, K. R. \& Yahel, G. Prey taxonomy rather than size determines salp diets. Limnol. Oceanogr. 64, 1996-2010 (2019)

55. Henschke, N., Everett, J. D., Richardson, A. J. \& Suthers, I. M. Rethinking the role of salps in the ocean. TRENDS Ecol. Evolution 31, 720-733 (2016).

56. Stone, J. P. \& Steinberg, D. K. Long-term time-series study of salp population dynamics in the Sargasso Sea. Mar. Ecol. Prog. Ser. 510, 111-127 (2014).

57. Stone, J. P. \& Steinberg, D. K. Salp contributions to vertical carbon flux in the Sargasso Sea. Deep Sea Res. I 113, 90-100 (2016).

58. Michaels, A. \& Knap, A. Overview of the U.S. JGOFS Bermuda Atlantic Timeseries Study and the Hydrostation S program. Deep-Sea Res. II 43, 157-198 (1996)

59. Steinberg, D. K. et al. Overview of the US JGOFS Bermuda Atlantic Timeseries Study (BATS): a decade-scale look at ocean biology and biogeochemistry. Deep-Sea Res. II 48, 1405-1447 (2001).

60. Knap, A. et al. BATS Methods Manual Version 4. (U.S. JGOFS Planning Office, Woods Hole, 1997).

61. Lohrenz, S. E. et al. Seasonal variability in primary production and particle-flux in the Northwestern Sargasso Sea-United-States Jgofs Bermuda Atlantic TimeSeries Study. Deep-Sea Res. Part a-Oceanographic Res. Pap. 39, 1373-1391 (1992).

62. Michaels, A. et al. Seasonal patterns of ocean biogeochemistry at the U.S. JGOFS Bermuda Atlantic Time-series study site. Deep-Sea Res. 41, 1013-1038 (1994).

63. Bates, N., Michaels, A. \& Knap, A. Seasonal and interannual variability of the oceanic carbon dioxide system at the U.S. JGOFS Bermuda Atlantic Timeseries Site. Deep Sea Res. II 43, 347-383 (1996).

64. Ducklow, H., Steinberg, D. K. \& Buesseler, K. O. Upper ocean carbon export and the biological pump. Oceanography 14, 50-58 (2001).

65. Sprintall, J. \& Tomczak, M. Evidence of the barrier layer in the surface layer of the tropics. J. Geophys. Res. - Oceans 97, 7305-7316 (1992).

66. Dore, J. et al. Freezing as a method of sample preservation for the analysis of dissolved inorganic nutrients in seawater. Mar. Chem. 53, 173-185 (1996).

67. Karl, D. M. \& Tien, G. MAGIC: a sensitive and precise method for measuring dissolved phosphorus in aquatic environments. Limnol. Oceanogr. 37, 105-116 (1992)

68. Rimmelin, P. \& Moutin, T. Re-examination of the MAGIC method to determine low orthophosphate concentration in seawater. Anal. Chim. Acta 548, 174-182 (2005).

69. Lomas, M. W. et al. Increased ocean carbon export in the Sargasso Sea is countered by its enhanced mesopelagic attenuation. Biogeosciences 7, 57-70 (2010).

70. Knauer, G., Martin, J. \& Bruland, K. Fluxes of particulate carbon, nitrogen, and phosphorus in the upper water column of the northeast Pacific. Deep-Sea Res. 26, 97-108 (1979).

71. Inomura, K. et al. A mechanistic model of macromolecular allocation, elemental stoichiometry, and growth rate in phytoplankton. Front. Microbiol. 11, https://doi.org/10.3389/fmicb.2020.00086 (2020).

72. Garcia, N., Bonachela, J. A. \& Martiny, A. C. Interactions between growthdependent changes in cell size, nutrient supply and cellular elemental stoichiometry of marine Synechococcus. ISME J. 10, 2715-2724 (2016).

73. Westberry, T. K., Behrenfeld, M. J., Siegel, D. A. \& Boss, E. Carbon-based primary productivity modeling with vertically resolved photoacclimation. Glob. Biogeochem. Cycle 22, GB2024 (2008).

74. Kamykowski, D., Zentara, J. S., Morrison, J. M. \& Switzer, A. C. Dynamic global patterns of nitrate, phosphate, silicate, and iron availability and phytoplankton community composition from remote sensing data. Glob. Biogeochem. Cycle 16, 25-21 (2002).

\section{Acknowledgements}

The authors acknowledge and thank the numerous principal investigators, researchers, and technicians who have contributed to the BATS time-series project since its inception Additional thanks go to the officers and crew of the R/V Weatherbird I, R/V Weatherbird II, and R/V Atlantic Explorer. NSF funding is acknowledged for M.W.L. (OCE-1756054), D.K.S. (OCE-1756312), and N.R.B. and R.J.J. (OCE-1756105). This is contribution 4076 of the Virginia Institute of Marine Science, William \& Mary.

\section{Author contributions}

M.W.L. conducted the analyses and wrote the first draft of the manuscript. R.J.J. assisted with providing data. T.T. contributed the results of and text describing the phytoplankton C:P model. D.K.S., N.R.B., R.J.J., and T.T. edited initial and revised drafts of the manuscript.

\section{Competing interests}

The authors declare no competing interests. 


\section{Additional information}

Supplementary information The online version contains supplementary material available at https://doi.org/10.1038/s41467-022-28842-3.

Correspondence and requests for materials should be addressed to Michael W. Lomas.

Peer review information Nature Communications thanks the anonymous reviewers for their contribution to the peer review of this work.

Reprints and permission information is available at http://www.nature.com/reprints

Publisher's note Springer Nature remains neutral with regard to jurisdictional claims in published maps and institutional affiliations. (c) (P) Open Access This article is licensed under a Creative Commons Attribution 4.0 International License, which permits use, sharing, adaptation, distribution and reproduction in any medium or format, as long as you give appropriate credit to the original author(s) and the source, provide a link to the Creative Commons license, and indicate if changes were made. The images or other third party material in this article are included in the article's Creative Commons license, unless indicated otherwise in a credit line to the material. If material is not included in the article's Creative Commons license and your intended use is not permitted by statutory regulation or exceeds the permitted use, you will need to obtain permission directly from the copyright holder. To view a copy of this license, visit http://creativecommons.org/ licenses/by/4.0/.

(C) The Author(s) 2022 